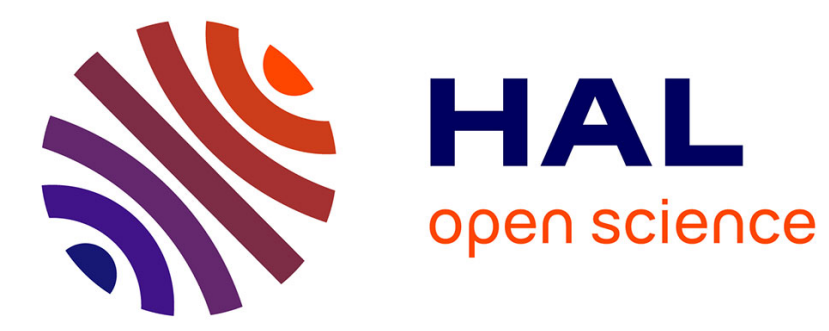

\title{
Spectral element computation of high-frequency leaky modes in three-dimensional solid waveguides
}

Fabien Treyssede

\section{To cite this version:}

Fabien Treyssede. Spectral element computation of high-frequency leaky modes in threedimensional solid waveguides. Journal of Computational Physics, 2016, 314, pp.341-354. 10.1016/j.jcp.2016.03.029 . hal-01346113

\section{HAL Id: hal-01346113 \\ https://hal.science/hal-01346113}

Submitted on 18 Jul 2016

HAL is a multi-disciplinary open access archive for the deposit and dissemination of scientific research documents, whether they are published or not. The documents may come from teaching and research institutions in France or abroad, or from public or private research centers.
L'archive ouverte pluridisciplinaire HAL, est destinée au dépôt et à la diffusion de documents scientifiques de niveau recherche, publiés ou non, émanant des établissements d'enseignement et de recherche français ou étrangers, des laboratoires publics ou privés. 


\title{
Spectral element computation of high-frequency leaky modes in three-dimensional solid waveguides
}

\author{
F. Treyssède ${ }^{\mathrm{a}, *}$ \\ ${ }^{a}$ LUNAM Université, IFSTTAR, GERS, GeoEND, F-44340 Bouguenais, France
}

\begin{abstract}
A numerical method is proposed to compute high-frequency low-leakage modes in structural waveguides surrounded by infinite solid media. In order to model arbitrary shape structures, a waveguide formulation is used, which consists of applying to the elastodynamic equilibrium equations a space Fourier transform along the waveguide axis and then a discretization method to the cross-section coordinates. However several numerical issues must be faced related to the unbounded nature of the cross-section, the number of degrees of freedom required to achieve an acceptable error in the high-frequency regime as well as the number of modes to compute. In this paper, these issues are circumvented by applying perfectly matched layers (PML) along the cross-section directions, a high-order spectral element method for the discretization of the cross-section, and an eigensolver shift suited for the computation of low-leakage modes. First, computations are performed for an embedded cylindrical bar, for which literature results are available. The proposed PML waveguide formulation yields good agreement with literature results, even in the case of weak impedance contrast. Its performance with high-order spectral elements is assessed in terms of convergence and accuracy and compared to traditional low-order finite elements. Then, computations are performed for an embedded square bar. Dispersion curves exhibit strong similarities with cylinders. These results show that the properties of low-leakage modes observed in cylindrical bars can also occur in other types of geometry.
\end{abstract}

Keywords:

\section{Introduction}

The modeling of structural waveguides is essential to understand the multimodal and dispersive propagation of guided waves in solids. A typical application of elastic guided waves is non-destructive evaluation (NDE) methods. The knowledge of modal properties of guided waves is necessary to optimize inspection systems by selecting the most suitable propagation modes for NDE, that is to say, the less dispersive and less attenuated modes. For canonical geometries (plates and cylinders), waveguides can be modeled thanks to analytical methods based on the Thomson-Haskell method or the global matrix method $[1,2]$ to provide modal characteristics as a function of frequency (dispersion curves).

Waveguides can be classified into two categories: closed waveguides (of bounded cross-section) and open waveguides (of unbounded cross-section). Of particular interest in this paper is the latter category, which includes: buried or embedded structures, partially buried waveguides and surface waveguides. Open waveguides are widely encountered in civil engineering. As typical applications, one can cite the NDE of steel rock bolts and bridge cables [3, 4] or buried pipes. Such structures are elongated and buried into an external medium (e.g. grout, concrete, soil), which can usually be considered as unbounded in the transverse direction.

Numerical methods are required for the modeling of arbitrary shape waveguides. One powerful numerical method consists of assuming the axial dependence of acoustic fields in the equations to be solved, and then

\footnotetext{
* Corresponding author

Email address: fabien.treyssede@ifsttar.fr (F. Treyssède)
} 
to apply a discretization method (usually the finite element method (FEM)) to the remaining coordinates, i.e. the cross-section.

As far as closed elastic waveguides are concerned, this method has been widely applied under various names, among which: semi-analytical finite element (SAFE) method [5, 6, 7], waveguide finite element method [8, 9], strip element method [10] or more recently the so-called scaled boundary finite element method (SBFEM) [11, 12]. These names indeed all refer to the same theoretical formulation, which will be referred to as waveguide formulation throughout this paper. The SBFEM uses a somehow different formalism to derive the equations but yields the same quadratic eigenproblem and elementary matrices as the other methods (the SBFEM actually differs only in the interpolation functions and the eigensystem linear form chosen to solve the problem).

With open waveguides, numerical methods must yet face difficulties due to the unbounded nature of the cross-section. This issue is enhanced by the intrinsic transverse growth to infinity of leaky modes, well-known in electromagnetism (see Ref. [13] for instance). To circumvent these difficulties, the waveguide formulation must be combined with other techniques.

An additional numerical difficulty is that the less attenuated modes in open elastic waveguides usually occur in a very high frequency regime $[3,4]$. This phenomenon is due to radiation losses, greater in a low-frequency regime, as opposed to material viscoelastic losses (which increases with frequency). Since the modal density increases with frequency, a large number of modes should be computed to identify these high-frequency low-leakage modes. Moreover their computation with the traditional FEM, using low-order elements, would require a very fine discretization involving a huge number of degrees of freedom (dofs).

For modeling open waveguides, one of the simplest numerical procedures consists of creating artificial viscoelastic layers in the surrounding medium for absorbing waves $[14,15]$. Waveguide formulations combined with the boundary element method have been recently developed to model three-dimensional elastic waveguides embedded in a solid [16, 17] or in a fluid [18]. The boundary element method avoids the discretization of the unbounded medium but yields a highly nonlinear eigenproblem, quite difficult to solve. Gravenkamp et al. [19] have proposed a simplified boundary condition, namely the dashpot boundary condition. This dashpot condition is usually no longer accurate for low frequency or for a low contrast of acoustic impedance. Similarly to the boundary element method, Hayashi et al. [20] have applied an exact radiation condition for two-dimensional plates and have successfully transformed the resulting nonlinear eigenproblem into a linear one. This transformation is yet only applicable to fluid external media. Besides, the extension to three-dimensional waveguides requires far-field approximations [21].

An alternative technique consists of using perfectly matched layers (PML). In addition to providing a linear eigenproblem in a straightforward manner, the PML approach remains applicable even for low contrast media. This technique has been applied to electromagnetic waveguides [22, 23], scalar waveguide problems $[24,25]$ and more recently to elastic waveguides [26, 27]. From a mathematical point of view, this approach turns out to be relevant because both leaky modes and PML can be defined through analytic continuations. Moreover, the convergence of the method for computing resonances in open systems has been proved for scalar wave problems $[28,29]$. In practice, the user can adjust PML parameters (thickness for instance) to check convergence towards physical solutions. Conversely as far as the dashpot boundary condition and artificial viscoelastic layers are concerned, theoretical explanations are still missing about the expected accuracy or convergence of solutions, in particular when the contrast of impedance becomes weak [30] (a comparison with these methods, although simple to implement, is left beyond the scope of this paper). One drawback with PML is that part of the exterior domain needs to be meshed, which may increase the computational cost compared to boundary element methods. Nevertheless, the thickness of the absorbing region can be greatly reduced compared to viscoelastic layers [27] thanks to the perfectly matched property.

In fact, the main drawback of the PML waveguide formulation is that the absorbing region leads to the existence of modes resonating mainly inside the PML. These modes can be viewed as discrete sets of two radiation mode continua in elastic problems [26]. In practice, solving the eigenproblem can lead to the computation of many radiation modes, of less interest for NDE applications, for only a limited number of leaky modes. And the computation cost of eigensolvers particularly increases with the number of modes to calculate. 
The goal of this paper is to propose a numerical method for the computation of high-frequency leaky modes in open solid waveguides. To do so, the waveguide formulation is combined with a PML technique. The resulting approach leads to a nonstandard eigenvalue problem which is quadratic and not self-adjoint. The cross-section is discretized with high-order spectral elements. Their performance for solving this specific eigenproblem is assessed in terms of convergence and accuracy and compared to traditional low-order finite elements. As expected, using high-order spectral elements enables to significantly reduce the number of dofs needed in the high-frequency regime to achieve a given discretization error. To circumvent the problem of calculating many modes, a property specific to low attenuation modes in embedded waveguides is used. In cylindrical structures, it has been found that low-leakage modes are of compressional type with phase velocities close to the longitudinal bulk velocity of the core [3]. The numerical results obtained in the present paper shows that this property still holds for other types of geometry. The computation of eigenvalues is hence centered around the wavenumber of bulk longitudinal waves, which allows to drastically reduce the number of modes to compute.

In this paper, three-dimensional waveguides are considered. The case of two-dimensional waveguides is less critical since the modal problem is one-dimensional and discretized with line elements, yielding rather fast computations even in a high-frequency regime.

\section{Numerical method}

\subsection{Open waveguide formulation with $P M L$}

Linear elastic materials are assumed. The time-harmonic dependence of acoustic fields is chosen as $e^{-\mathrm{i} \omega t}$. Taking advantage of the symmetry of the stress tensor, the three-dimensional equilibrium equations of elastodynamics can be written as:

$$
\tilde{\mathbf{L}}^{\mathrm{T}} \tilde{\boldsymbol{\sigma}}+\tilde{\rho} \omega^{2} \tilde{\mathcal{U}}=\mathbf{0}
$$

where $\tilde{\mathcal{U}}$ is the acoustic displacement vector field and $\tilde{\boldsymbol{\sigma}}$ is the Voigt notation representation of the stress tensor ( $\tilde{\boldsymbol{\sigma}}$ is a six-dimensional vector). The stress-strain relation is given by $\tilde{\boldsymbol{\sigma}}=\tilde{\mathbf{C}} \tilde{\boldsymbol{\epsilon}}$, with $\tilde{\mathbf{C}}$ and $\tilde{\boldsymbol{\epsilon}}$ denoting the matrix of material properties and the strain vector respectively. The superscript $\mathrm{T}$ denotes the matrix transpose. The operator $\tilde{\mathbf{L}}$ is defined by $\tilde{\mathbf{L}}=\mathbf{L}_{i} \frac{\partial}{\partial \tilde{x}_{i}}$, where the Einstein summation convention is used for $i$ $(i=1,2,3)$ and:

$$
\mathbf{L}_{1}=\left[\begin{array}{lll}
1 & 0 & 0 \\
0 & 0 & 0 \\
0 & 0 & 0 \\
0 & 1 & 0 \\
0 & 0 & 1 \\
0 & 0 & 0
\end{array}\right], \quad \mathbf{L}_{2}=\left[\begin{array}{lll}
0 & 0 & 0 \\
0 & 1 & 0 \\
0 & 0 & 0 \\
1 & 0 & 0 \\
0 & 0 & 0 \\
0 & 0 & 1
\end{array}\right], \quad \mathbf{L}_{3}=\left[\begin{array}{lll}
0 & 0 & 0 \\
0 & 0 & 0 \\
0 & 0 & 1 \\
0 & 0 & 0 \\
1 & 0 & 0 \\
0 & 1 & 0
\end{array}\right]
$$

Let us denote $x_{3}$, the axis of the waveguide. In order to account for the unbounded nature of the crosssection, a PML is applied along the cross-section real variables $x_{\alpha}(\alpha=1,2)$. This is achieved by extending the elastodynamic equilibrium equations into complex coordinates $\tilde{x}_{\alpha}\left(x_{\alpha}\right)$ in order to absorb waves in the surrounding medium, with:

$$
\tilde{x}_{\alpha}=\int_{0}^{x_{\alpha}} \gamma_{\alpha}(\zeta) d \zeta
$$

where $\gamma_{\alpha}$ is a complex-valued function of $x_{\alpha}$ in the absorbing region defined by $d_{\alpha}<\left|x_{\alpha}\right| \leq d_{\alpha}+h_{\alpha}$. The user-defined parameters $h_{\alpha}$ and $d_{\alpha}$ denote the PML thickness and the position of the interface between the PML and the physical region, along the $x_{\alpha}$-axis (see Fig. 1a). The function $\gamma_{\alpha}$ is also user-defined and must satisfy: $\gamma_{\alpha}\left(x_{\alpha}\right)=1$ for $\left|x_{\alpha}\right| \leq d_{\alpha}$, and $\operatorname{Im}\left\{\gamma_{\alpha}\left(x_{\alpha}\right)\right\}>0$ for $\left|x_{\alpha}\right|>d_{\alpha}$.

First, a change of variable is performed to transform the equilibrium equations (1) back to real coordinates. From Eq. (3), the change of variables $\tilde{x}_{\alpha} \mapsto x_{\alpha}$, yields for any function $\tilde{f}$ :

$$
\frac{\partial \tilde{f}}{\partial \tilde{x}_{\alpha}}=\frac{1}{\gamma_{\alpha}} \frac{\partial f}{\partial x_{\alpha}}
$$


where $\tilde{f}\left(\tilde{x}_{1}\left(x_{1}\right), \tilde{x}_{2}\left(x_{2}\right), x_{3}\right)=f\left(x_{1}, x_{2}, x_{3}\right)$. In real coordinates, the strain-displacement relation is hence written as:

$$
\boldsymbol{\epsilon}=\left(\frac{1}{\gamma_{\alpha}} \mathbf{L}_{\alpha}(\cdot)_{, \alpha}+\mathbf{L}_{3}(\cdot)_{, 3}\right) \mathcal{U}
$$

where the Einstein summation's rule is used for cross-section coordinates with Greek indices $(\alpha=1,2)$. The notation $(\cdot)_{, i}$ denotes partial derivatives with respect to the real coordinates $x_{i}(i=1,2,3)$.

Then, the axial variable is separated into the elastodynamic equilibrium equations by expressing the acoustic fields with a harmonic dependence in $e^{\mathrm{i} k x_{3}}$, where $k$ is the axial wavenumber. The three-dimensional acoustic displacement vector field $\mathcal{U}$ can then be written as:

$$
\mathcal{U}\left(x_{1}, x_{2}, x_{3}\right)=\mathbf{u}\left(x_{1}, x_{2}\right) e^{\mathrm{i} k x_{3}}
$$

The discretization method, e.g. the FEM, is applied to the two-dimensional cross-section of the waveguide by interpolating the acoustic displacement $\mathbf{u}$ on each element $e$ as follows:

$$
\mathbf{u}^{e}(\xi, \eta)=\mathbf{N}^{e}(\xi, \eta) \mathbf{U}^{e}
$$

where the column vector $\mathbf{U}^{e}$ contains nodal displacements and $\mathbf{N}^{e}$ is a matrix of nodal interpolating functions on the element mapped to a square rectangle $[-1 ;+1] \times[-1 ;+1]$ in the reference plane $(\xi, \eta)$. The Jacobian matrix of the transformation from $\left(x_{1}, x_{2}\right)$ to $(\xi, \eta)$ will be denoted by $\mathbf{J}^{e}(\xi, \eta)$.

From Eqs. (4)-(7), the weak form of the equilibrium equations of elastodynamics integrated on the cross-section $S\left(d S=d x_{1} d x_{2}\right)$ finally yields the following eigenvalue problem:

$$
\left\{\mathbf{K}_{1}-\omega^{2} \mathbf{M}+\mathrm{i} k\left(\mathbf{K}_{2}-\mathbf{K}_{2}^{\mathrm{T}}\right)+k^{2} \mathbf{K}_{3}\right\} \mathbf{U}=\mathbf{0}
$$

with the elementary matrices:

$$
\begin{gathered}
\mathbf{K}_{1}^{e}=\int_{-1}^{+1} \int_{-1}^{+1}\left(\mathbf{L}_{\alpha} \mathbf{N}_{, \alpha}^{e}\right)^{\mathrm{T}} \mathbf{C}\left(\mathbf{L}_{\beta} \mathbf{N}_{, \beta}^{e}\right) \frac{\gamma_{1} \gamma_{2}}{\gamma_{\alpha} \gamma_{\beta}}\left|\operatorname{det} \mathbf{J}^{e}\right| d \xi d \eta, \mathbf{M}^{e}=\int_{-1}^{+1} \int_{-1}^{+1} \rho \mathbf{N}^{e \mathrm{~T}} \mathbf{N}^{e} \gamma_{1} \gamma_{2}\left|\operatorname{det} \mathbf{J}^{e}\right| d \xi d \eta \\
\mathbf{K}_{2}^{e}=\int_{-1}^{+1} \int_{-1}^{+1}\left(\mathbf{L}_{\alpha} \mathbf{N}_{, \alpha}^{e}\right)^{\mathrm{T}} \mathbf{C} \mathbf{L}_{3} \mathbf{N}^{e} \frac{\gamma_{1} \gamma_{2}}{\gamma_{\alpha}}\left|\operatorname{det} \mathbf{J}^{e}\right| d \xi d \eta, \mathbf{K}_{3}^{e}=\int_{-1}^{+1} \int_{-1}^{+1} \mathbf{N}^{e \mathrm{~T}} \mathbf{L}_{3}^{\mathrm{T}} \mathbf{C L}_{3} \mathbf{N}^{e} \gamma_{1} \gamma_{2}\left|\operatorname{det} \mathbf{J}^{e}\right| d \xi d \eta
\end{gathered}
$$

Additional details on the elastic PML waveguide formulation can be found in Ref. [27].

\subsection{Spectral elements}

The PML waveguide formulation can be further enhanced by discretizing the cross-section with highorder spectral elements instead of traditional low-order finite elements.

The SEM was originally applied to transient problems in fluid dynamics [31] and later in elastic wave propagation (see for instance Refs. $[32,33]$ ). The SEM is indeed able to deliver low dispersion error and is particularly attractive for fully three-dimensional problems or high-frequency wave problems, which require intensive computations.

Like the FEM, the SEM uses Lagrange interpolation polynomials. The main difference is that the SEM interpolation is based on Gauss-Lobatto-Legendre (GLL) nodes instead of equispaced nodes, together with the use of GLL quadrature rules instead of the Gauss-Legendre quadrature. For a quadrangle spectral element of order $r$, Eq. (7) can be rewritten as follows:

$$
\mathbf{u}^{e}(\xi, \eta)=\sum_{i=1}^{r+1} \sum_{j=1}^{r+1} h_{i}^{e}(\xi) h_{j}^{e}(\eta) \mathbf{u}^{e}\left(\xi_{i}, \eta_{j}\right)
$$

where the $h_{i}$ 's $(i=1, \ldots, r+1)$ denotes the one-dimensional Lagrange interpolants of order $r$ :

$$
h_{i}^{e}(\xi)=\prod_{j=1, j \neq i}^{r+1} \frac{\xi-\xi_{j}}{\xi_{i}-\xi_{j}}
$$




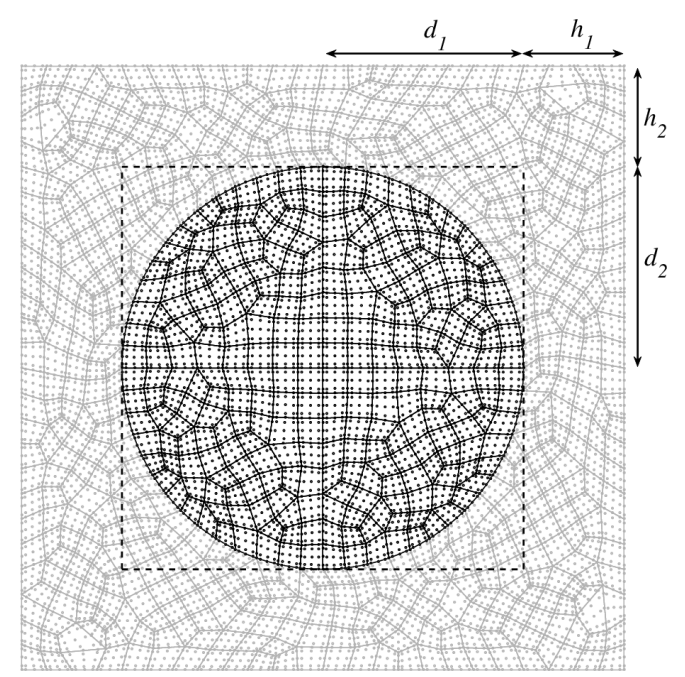

(a)

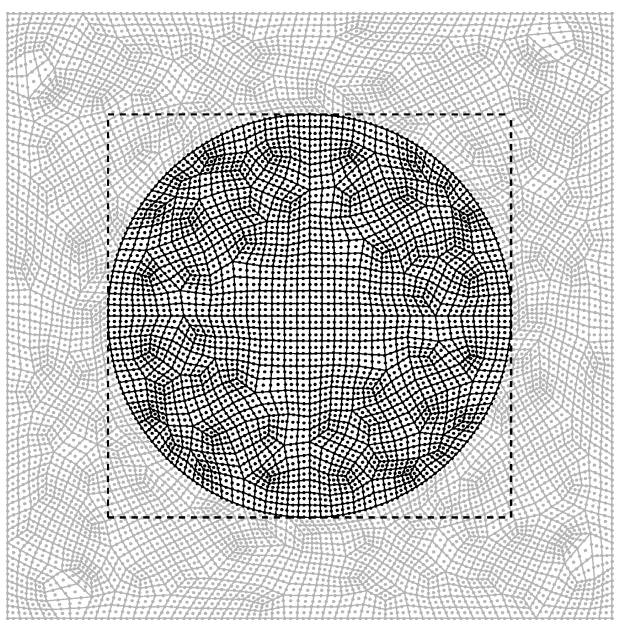

(b)

Figure 1: Discretization of an embedded cylindrical waveguide with: (a) 4th order spectral elements, (b) traditional 2nd order finite elements (39267 dofs). Black: cylindrical bar (steel), gray: external medium (cement grout). Dashed line: PML interface. The 2nd order mesh results from a subdivision of each quadrangle of the 4th order mesh so that both meshes yield the same number of nodes and dofs.

and the $\xi_{i}$ 's denotes the $r+1$ GLL points along the direction $\xi$, defined by the roots of $\left(1-\xi^{2}\right) P_{r}^{\prime}(\xi)=0$ with $P_{r}(\xi)$ denoting the Legendre polynomial of degree $r$. The same definition holds for the $\eta_{j}$ 's, the $r+1$ GLL points along the direction $\eta$. The GLL weights $\omega_{i}(i=1, \ldots, r+1)$ used for the quadrature rule are given by:

$$
\omega_{1}=\omega_{r+1}=\frac{2}{r(r+1)}, \quad \omega_{i}=\frac{2}{r(r+1) P_{r}\left(\xi_{i}\right)^{2}} \quad(i=2, \ldots, r)
$$

with $\xi_{1}=-1$ and $\xi_{r+1}=+1$. The integrals in Eq. (9) are then evaluated from the numerical quadrature:

$$
\int_{-1}^{+1} \int_{-1}^{+1} f(\xi, \eta) d \xi d \eta \simeq \sum_{i}^{r+1} \sum_{j}^{r+1} \omega_{i} \omega_{j} f\left(\xi_{i}, \eta_{j}\right)
$$

Figure 1a shows a typical example of SEM mesh used in this paper with the PML waveguide formulation. Note that positioning the internal nodes at the GLL quadrature points is crucial to ensure an order of convergence of the approximated eigenvalues with a rate of $h^{2 r}$ [34] (where $h$ is a measure of the size of elements). Conversely, the FEM traditionally uses equispaced nodes and suffers from Runge's phenomenon and a rapid increase of condition numbers as the order of interpolation increases (see for instance Refs. [35, $36])$.

\subsection{Structure of the eigenvalue problem}

With the SEM, the interpolation nodes coincide with the quadrature points so that:

$$
h_{i}^{e}\left(\xi_{j}\right)=h_{i}^{e}\left(\eta_{j}\right)=\delta_{i j}
$$

This naturally leads to a diagonal mass matrix $\mathbf{M}$ and also enables to reduce the computation cost of the derivatives $(\cdot)_{, \xi}$ and $(\cdot)_{, \eta}[37]$.

For transient problems, the diagonalization of the mass matrix drastically reduces the computation cost. Yet for the eigenvalue problem given by Eq. (8), this property could only be useful provided that $\omega$ be the eigenvalue and that $k$ be then set to a fixed real value. Such an approach is only applicable to lossless waveguides in practice. 
In this paper, our interest focuses on leaky modes, which have complex eigenvalues because of radiation loss. Given $\omega$, Eq. (8) is a quadratic eigenvalue problem with respect to the eigenvalue $k$. This quadratic problem can be transformed to a linear eigenproblem as follows:

$$
(\mathbf{A}-k \mathbf{B}) \hat{\mathbf{U}}=0
$$

with:

$$
\mathbf{A}=\left[\begin{array}{cc}
\mathbf{0} & \mathbf{I} \\
-\left(\mathbf{K}_{1}-\omega^{2} \mathbf{M}\right) & -\mathrm{i}\left(\mathbf{K}_{2}-\mathbf{K}_{2}^{\mathrm{T}}\right)
\end{array}\right], \quad \mathbf{B}=\left[\begin{array}{cc}
\mathbf{I} & \mathbf{0} \\
\mathbf{0} & \mathbf{K}_{3}
\end{array}\right], \quad \hat{\mathbf{U}}=\left[\begin{array}{c}
\mathbf{U} \\
k \mathbf{U}
\end{array}\right]
$$

Other linear forms are possible [38]. In this paper, the eigensystem (15) is solved with the ARPACK library [39], appropriate for large sparse matrices and based on the implicitly restarted Arnoldi method.

Without PML, the above linear form has the advantage of yielding a Hermitian matrix $\mathbf{B}$ that is wellsuited for an efficient treatment by the library [40]. However with PML and FEM, $\mathbf{K}_{3}$ is a symmetric banded sparse matrix, non-Hermitian, and so is $\mathbf{B}$.

With PML and SEM, the non-Hermitian matrix $\mathbf{K}_{3}$ indeed becomes block diagonal with blocks of dimension three, as shown in Appendix A. The matrix $\mathbf{K}_{3}$ can thus be easily inverted, as well as $\mathbf{B}$, so that the eigenvalue problem given by Eqs. (15)-(16) can be readily recast into a standard eigenvalue problem, $\left(\mathbf{B}^{-1} \mathbf{A}-k \mathbf{I}\right) \hat{\mathbf{U}}=0$, less consuming in terms of memory and more efficiently solved by the eigensolver. Note that the matrix $\mathbf{K}_{3}$ is diagonal in the case of isotropic materials. It also remains diagonal for transversely isotropic materials as shown in Appendix A.

\subsection{Eigensolver shift}

With the ARPACK library, a specified number of eigenvalues is looked for around a user-defined shift. A property specific to low attenuation modes in embedded waveguides is used to circumvent the problem of calculating too many modes. In cylindrical structures, low-leakage modes are of compressional type [3]. These modes, usually labelled as $L(0, n)$, are of interest for NDE because they combine low axial attenuation, low dispersion as well as high energy velocity at some particular frequencies. These specific frequencies occur close to the longitudinal bulk velocity of the core, denoted as $c_{l_{0}}$. In order to find these modes, the computation of eigenvalues can hence be centered around the wavenumber shift $k_{0}=\omega / c_{l_{0}}$. In this way, the user-defined number of modes to compute can be greatly reduced. This avoids the computation of many radiation or highly attenuated leaky modes, of less interest in practice.

Figure 2 shows an example of eigenspectrum computed with the PML waveguide formulation. One considers a steel cylindrical bar embedded in cement grout (a full description of this test case is given in Sec. 3.1). The spectrum is dimensionless and plotted for $-k^{2} a^{2}$, where $a$ is a characteristic length defined later. The dimensionless frequency is set to $\omega a / c_{s_{0}}=10$, with $c_{s_{0}}$ denoting the shear bulk velocity of the core. Two kinds of modes occur. The first kind corresponds to radiation modes. These modes can be interpreted as the discretization of two continua (branch cuts) emanating from the branch points $-\omega^{2} a^{2} / c_{l_{\infty}}^{2}$ and $-\omega^{2} a^{2} / c_{s_{\infty}}^{2}$, where $c_{l_{\infty}}$ and $c_{s_{\infty}}$ denote the bulk longitudinal and shear wave velocities of the external medium. As shown in Ref. [27], the radiation poles are approximately located along straight lines rotated in the complex plane from the real axis with an angle equal to $-2 \arg \left(d_{\alpha}+h_{\alpha} \hat{\gamma}_{\alpha}\right)$ (the hat is used to denote the mean value inside the PML). Leaky modes are then revealed in the region covered by the rotation of radiation modes.

As observed in Fig. 2, the wavenumber shift $k_{0} a=\omega a / c_{l_{0}}$ allows to focus the computation of eigenvalues on the leaky mode region where low-leakage modes of compressional type occur. Setting a sufficiently small number of modes to compute around $k_{0}$ avoids the computation of radiation modes.

\section{Results}

\subsection{Parameters}

One considers a viscoelastic steel cylindrical bar embedded in a viscoelastic cement grout. Both materials are isotropic. Their properties are given in Table 1. This test case is taken from Refs. [2, 3, 41]. With 


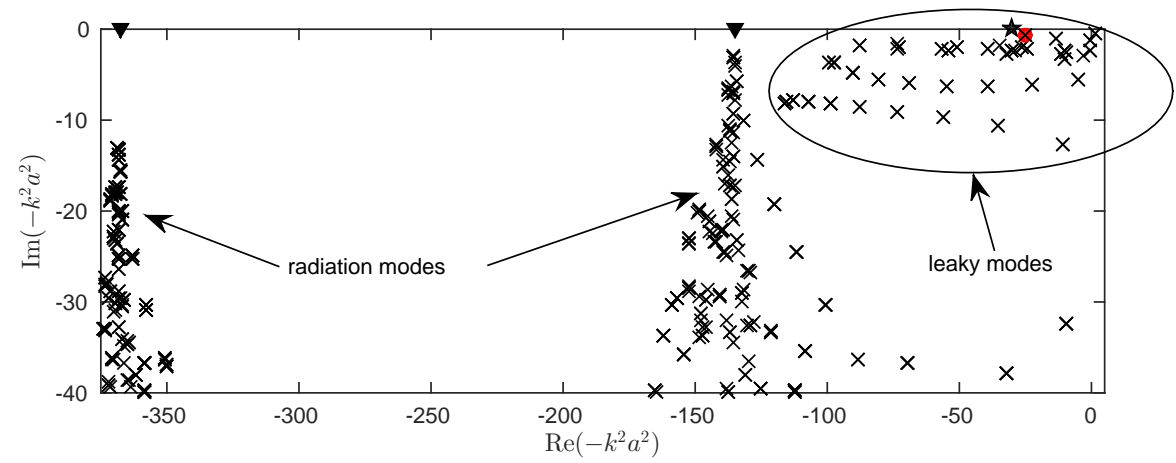

Figure 2: Example of eigenspectrum (steel bar in cement grout, $\omega a / c_{s_{0}}=10$ ). Crosses: poles computed with the PML waveguide formulation. The pole highlighted in red is the low-leakage compressional mode (here, $\mathrm{L}(0,4)$ ). Triangles: theoretical branch points $\left(-\omega^{2} a^{2} / c_{s_{\infty}}^{2}, 0\right)$ and $\left(-\omega^{2} a^{2} / c_{l_{\infty}}^{2}, 0\right)$. Star: user-defined eigensolver shift $\left(-k_{0}^{2} a^{2}, 0\right)$.

\begin{tabular}{lccccc}
\hline \hline & $\begin{array}{c}\text { Density } \\
\text { Material }\end{array}$ & $\begin{array}{c}\text { Longitudinal } \\
\text { velocity } \\
\left(\mathrm{kg} / \mathrm{m}^{3}\right)\end{array}$ & $\begin{array}{c}\text { Shear } \\
c_{l}(\mathrm{~m} / \mathrm{s})\end{array}$ & $\begin{array}{c}\text { Longitudinal bulk } \\
c_{s}(\mathrm{~m} / \mathrm{s})\end{array}$ & $\begin{array}{c}\text { Shear bulk } \\
\text { wave attenuation }\end{array}$ \\
$\begin{array}{l}\beta_{l}(\mathrm{~Np} / \text { wavelength }) \\
\text { wave attenuation } \\
\beta_{s}(\mathrm{~Np} / \text { wavelength })\end{array}$ \\
Ceel & 7932 & 5960 & 3260 & 0.003 & 0.008 \\
Cast iron & 1600 & 2810 & 1700 & 0.043 & 0.1 \\
\hline \hline
\end{tabular}

Table 1: Material properties.

viscoelasticity, Young's modulus and Poissons ratio are complex and given by:

$$
E=\rho \tilde{c}_{s}^{2} \frac{3 \tilde{c}_{l}^{2}-4 \tilde{c}_{s}^{2}}{\tilde{c}_{l}^{2}-\tilde{c}_{s}^{2}}, \quad \nu=\frac{1}{2} \frac{\tilde{c}_{l}^{2}-2 \tilde{c}_{s}^{2}}{\tilde{c}_{l}^{2}-\tilde{c}_{s}^{2}}
$$

with the complex bulk velocities $\tilde{c}_{l}$ and $\tilde{c}_{s}$ :

$$
\tilde{c}_{l, s}=c_{l, s}\left(1+\mathrm{i} \frac{\beta_{l, s}}{2 \pi}\right)^{-1}
$$

where $\beta_{l}$ and $\beta_{s}$ denote the bulk wave attenuations in Neper per wavelength.

The following parabolic profile is adopted for both the real and the imaginary parts of the PML functions $\gamma_{\alpha}(\alpha=1,2)$ :

$$
\gamma_{\alpha}\left(x_{\alpha}\right)=\left\{\begin{array}{lll}
1 & \text { if } & \left|x_{\alpha}\right| \leq d_{\alpha} \\
1+3\left(\hat{\gamma}_{\alpha}-1\right)\left(\frac{\left|x_{\alpha}\right|-d_{\alpha}}{h_{\alpha}}\right)^{2} & \text { if } & \left|x_{\alpha}\right|>d_{\alpha}
\end{array},\right.
$$

where $\hat{\gamma}_{\alpha}$ is the mean value of $\gamma_{\alpha}$ in the direction $x_{\alpha}$ inside the PML and quantifies the PML absorption. In this paper, the PML parameters are set to: $\hat{\gamma}_{\alpha}=2+4 \mathrm{i}, h_{\alpha}=0.5 a$ and $d_{\alpha}=a$ with $a$ denoting the radius of the steel bar. A Dirichlet condition is applied at the PML exterior boundaries. Note that the PML interface is set as close as possible to the core in order to avoid spurious eigenvalues associated with large values of the norm of resolvent [26].

In this paper, the dispersion curves will be plotted both for the energy velocity and the attenuation. The attenuation in $\mathrm{dB} / \mathrm{m}$ is defined as $8.686 \operatorname{Im}(k)$. Following Ref. [42], the energy velocity of open waveguides can be defined by restricting the integration of fields on $S_{0}$, the cross-section of the core (i.e. excluding the unbounded surrounding region):

$$
v_{e}=\frac{\int_{S_{0}} \Pi \mathrm{d} S_{0}}{\int_{S_{0}}\left(E_{k}+E_{p}\right) \mathrm{d} S_{0}}
$$


where $\Pi$ denotes the time-averaged axial component of the Poynting vector, $E_{k}$ and $E_{p}$ are the time-averaged kinetic and potential energy respectively. These quantities can be post-processed in a straightforward manner from the matrices and solutions of the eigensystem (8) as follows [27]:

$$
\begin{aligned}
\int_{S_{0}} \Pi \mathrm{d} S_{0}= & \frac{\omega}{2} \operatorname{Im}\left\{\overline{\mathbf{U}}^{*}\left(\overline{\mathbf{K}}_{2}+\mathrm{i} k \overline{\mathbf{K}}_{3}\right) \overline{\mathbf{U}}\right\}, \int_{S_{0}} E_{k} \mathrm{~d} S_{0}=\frac{\omega^{2}}{4} \operatorname{Re}\left(\overline{\mathbf{U}}^{*} \overline{\mathbf{M}} \overline{\mathbf{U}}\right), \\
& \int_{S_{0}} E_{p} \mathrm{~d} S_{0}=\frac{1}{4} \operatorname{Re}\left\{\overline{\mathbf{U}}^{*}\left(\overline{\mathbf{K}}_{1}+\mathrm{i} k \overline{\mathbf{K}}_{2}-\mathrm{i} k^{*} \overline{\mathbf{K}}_{2}^{\mathrm{T}}+k^{*} k \overline{\mathbf{K}}_{3}\right) \overline{\mathbf{U}}\right\}
\end{aligned}
$$

The notation $\overline{(\cdot)}$ is used to denote the restriction of vectors and matrices to the dofs of the core.

The finite element meshes are built from the free software Gmsh [43], which allows generating high-order elements with equispaced nodes. The resulting equispaced nodes are then moved to GLL points thanks to an external in-house routine.

Keeping in mind the purpose of modeling arbitrary shape waveguides, the symmetry of cross-sections is not accounted for in this paper although it could have been used to further reduce the computational cost.

\subsection{Dispersion curves}

In order to plot the dispersion curves, the eigenvalue problem (15) is solved for 400 evenly spaced frequencies up to the frequency-radius of $26 \mathrm{MHz}-\mathrm{mm}$, i.e. a maximum dimensionless frequency $\omega a / c_{s_{0}}$ equal to 50. Note that the function $\gamma_{\alpha}$ given by Eq. (19) is independent of $\omega$, which avoids the calculation of matrices at each frequency.

The geometry is discretized with the SEM of order 4 (25-node quadrangles), which is a widespread choice for meshing arbitrary shape geometry (see for instance [44]). A meshing criterion of at least 5 points per wavelength is satisfied. The minimum wavelength in steel is its shear wavelength at the maximum frequency, $\lambda_{s} / a=c_{s} / f a \simeq 1 / 8$. Hence, satisfying a 5 point per wavelength criterion roughly requires 8 spectral elements of order 4 along the bar radius. Figure 1a exhibits the spectral element mesh used in the analysis.

For comparison, the problem is also solved by discretizing the geometry with the FEM of order 2 (ninenode quadrangles). The corresponding mesh, shown in Fig $1 \mathrm{~b}$, is generated by subdividing each quadrangle of the 4th spectral element mesh into 4 quadrangles. This enables to generate exactly the same number of nodes and dofs in both meshes (here, 39267 dofs).

As already explained in Sec. 2.4, the eigenvalue search is centered around the shift $k_{0}=\omega / c_{l_{0}}$. In practice, only 25 computed modes were necessary at each frequency to find the high-frequency low-leakage modes, namely the $L(0, n)$ modes.

Figure 3 shows the energy velocity and attenuation dispersion curves computed with the meshes of Fig. 1. The attenuation curves obtained with the 4 th order SEM are in agreement over the whole frequency range with those of Ref. [2], obtained from an analytical approach based on a global matrix method (software Disperse [45]), while some differences are found with the energy velocity for the highest frequencies. This is due to the fact that the eigenvectors, required to post-process the energy velocity, are numerically determined with a lower accuracy than the eigenvalues [34]. As found in Refs. [3, 41], it can be observed that the attenuation minima of the $L(0, n)$ modes almost coincide with their energy velocity maxima. Besides, the successive attenuation minima decrease up to $23 \mathrm{MHz}-\mathrm{mm}$ and then increase slowly. This phenomenon is explained by the counteracting effects of viscoelasticity and leakage on guided waves (the former tends to increase the attenuation as the frequency increases and conversely for the latter).

As expected, the accuracy of results computed with the traditional 2nd order FEM deteriorates in the high-frequency regime. It is well-known that at least four 2nd order elements per wavelength are necessary to get an acceptable convergence of numerical results, that is to say, 9 points per wavelength. Here, such a criterion is satisfied up to the frequency $13 \mathrm{MHz}-\mathrm{mm}$ and yields a close agreement with the 4th order SEM.

In Table 2, numerical values are quantitatively compared to those of Ref. [41], providing the minimum attenuation of a few modes and their corresponding frequency. Very good agreement is found. The less attenuated mode turns out to be $L(0,12)$ at $22.9 \mathrm{MHz}-\mathrm{mm}$. Discrepancies are expectedly greater with the 2nd order FEM, in particular for the frequency values. 


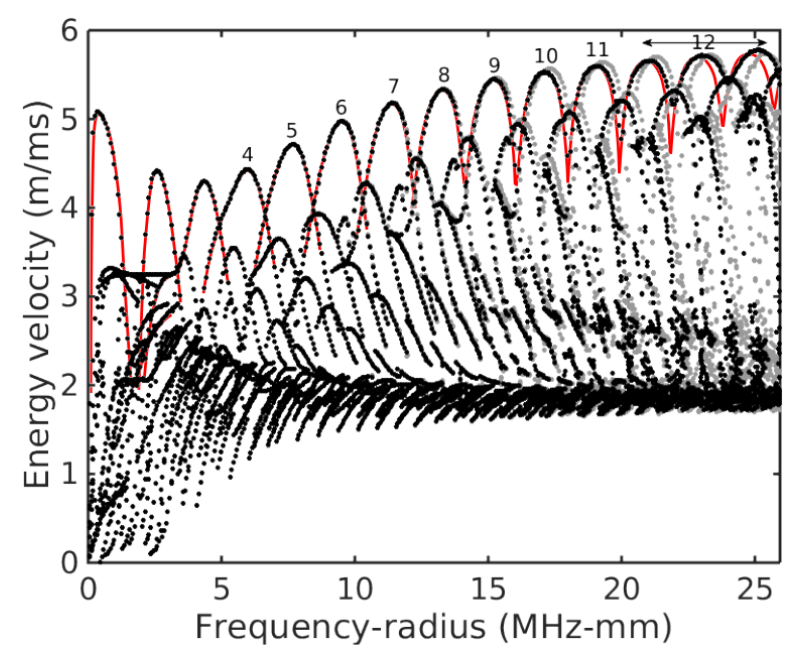

(a)

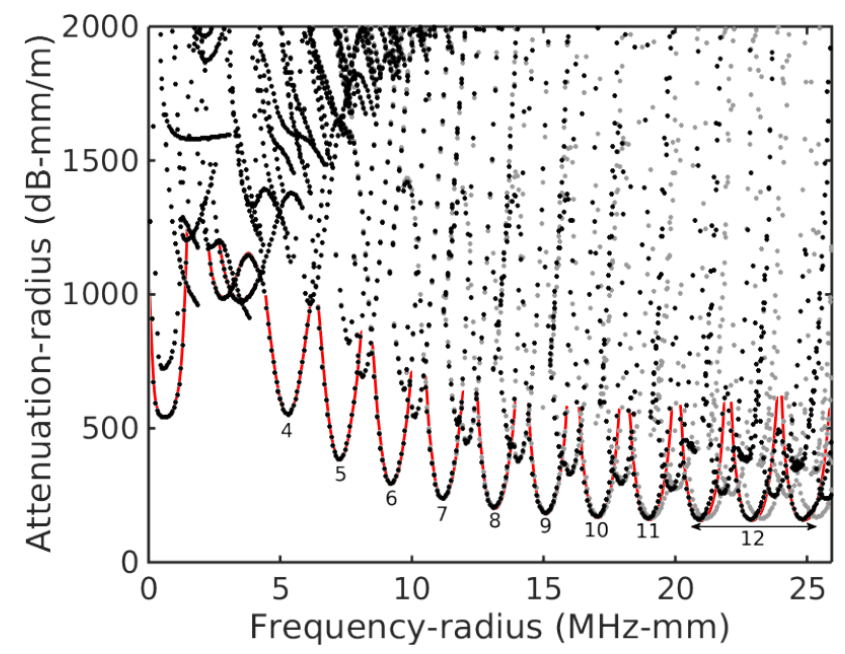

(b)

Figure 3: Dispersion curves of $L(0, n)$ modes computed with the meshes of Fig. 1 (39267 dofs): (a) energy velocity, (b) attenuation. Black: 4th order SEM results, gray: 2nd order FEM. Red continuous line: Pavlakovic's results [2]

\begin{tabular}{lcccc}
\hline \hline & \multicolumn{2}{c}{ Frequency-radius $(\mathrm{MHz}-\mathrm{mm})$} & \multicolumn{2}{c}{ Attenuation-radius $(\mathrm{dB}-\mathrm{mm} / \mathrm{m})$} \\
Mode & Disperse & Numerical & Disperse & Numerical \\
\hline$L(0,8)$ & 13.1 & $13.1(13.1)$ & 206 & $205(205)$ \\
$L(0,9)$ & 15.1 & $15.1(15.1)$ & 184 & $184(184)$ \\
$L(0,10)$ & 17.0 & $17.0(17.1)$ & 171 & $170(172)$ \\
$L(0,11)$ & 19.0 & $19.0(19.1)$ & 164 & $163(166)$ \\
$L(0,12)$ & 22.9 & $22.9(23.3)$ & 159 & $160(164)$ \\
\hline \hline
\end{tabular}

Table 2: Comparison between Disperse [41] and numerical results obtained with 4th order SEM at the attenuation minima of five modes. In parentheses: numerical results with 2nd order FEM. 


\begin{tabular}{lcccc}
\hline \hline Mesh & \# of nodes & \# of elements & \# of dofs & $h / a$ \\
\hline 1 & 721 & $40(160)$ & 2163 & $1 / 8$ \\
2 & 2881 & $168(672)$ & 8643 & $1 / 16$ \\
3 & 13089 & $792(3168)$ & 39267 & $1 / 32$ \\
4 & 54241 & $3336(13344)$ & 162723 & $1 / 64$ \\
\hline \hline
\end{tabular}

Table 3: Mesh characteristics used for convergence tests with 4th order SEM. $h / a$ is the dimensionless average distance between successive nodes inside the cylindrical bar. Characteristics are the same for 2nd order FEM meshes except the number of elements (in parentheses).

\subsection{Numerical convergence}

The numerical convergence of solutions is now assessed through $h$-refinement. Four meshes are considered by successively dividing by a factor 2 the average size of quadrangles. The characteristics of meshes are given in Table 3. The mesh used in the previous subsection for plotting the dispersion curves is Mesh 3. For a given mesh number, the number of nodes and dofs with 4th order elements is identical with 2nd order elements, because each 4th order quadrangle element has been divided into four 2nd order quadrangles similarly to the example given by Fig. 1 . As a consequence, the number of 2 nd order elements is exactly equal to four times the number of 4 th order elements.

The reference value $k_{r}$ is chosen as the numerical value computed with the finest mesh (mesh 4). The numerical convergence of the problem is assessed under a specified set of PML parameters. This allows to get rid of the effects of PML parameters on convergence. One recalls that PML parameters are user-defined and should be optimized for each mesh and frequency. Such an optimization is a well-known problem in the PML literature and is out of scope here. The PML parameters are those of Sec. 3.1 (they have shown to give satisfying results in Sec. 3.2).

The convergence curves are plotted for the wavenumber relative error, $\left|k-k_{r}\right| /\left|k_{r}\right|$, as a function of the mesh parameter $N=c_{s} / f h . N$ can be viewed as the number of grid steps per wavelength along one direction. The number of points (nodes) per wavelength is hence $N+1$. Two modes are considered at their respective minimum attenuation: the $L(0,8)$ mode at $13.1 \mathrm{MHz}-\mathrm{mm}\left(k_{r}=13.6121+0.0235 \mathrm{i}\right)$ and the $L(0,12)$ mode at $22.9 \mathrm{MHz}-\mathrm{mm}\left(k_{r}=24.0195+0.0184 \mathrm{i}\right)$. Figure 4 shows the convergence curves computed with both the 4th order SEM and the 2nd order FEM. As expected, the former exhibits a higher rate of convergence.

When $N$ is large enough, the convergence rates tend to the slope $s=-2 r$ ( $r$ being the element order), in agreement with the theoretical error found for symmetric eigenproblems [34]: $\left|k-k_{r}\right|=O\left(h^{2 r}\right)$. This asymptotic behavior can be clearly observed in Fig. 4a, as opposed to Fig. 4b where the value of $N$ is not large enough owing to the higher frequency considered. Note that with the 2nd order FEM, no convergence can be clearly observed for the $L(0,12)$ mode.

Also shown in Fig. 4 is the relative error for the imaginary part of the wavenumber, $\left|\operatorname{Im}(k)-\operatorname{Im}\left(k_{r}\right)\right| /\left|\operatorname{Im}\left(k_{r}\right)\right|$. The attenuation is a modal feature of great importance for NDE (it allows to estimate the maximum inspection distance). The assessment of its convergence and accuracy is thus of particular interest. With the 2nd order FEM, the convergence rate of the attenuation does not necessarily follow the theoretical slopes of convergence $s=-2 r$. This is somehow an expected result because the imaginary part of the wavenumber is several orders of magnitude lower than the real part. Yet with the 4th order SEM, the imaginary part nearly follows the same slope as $k$.

In addition to the convergence rate, the vertical position of curves is another feature of importance as it gives a measure of the accuracy of elements and allows to determine how small $h$ should be to reach an acceptable error. From Fig. 4, it can roughly be found that an accuracy of $0.01 \%$ is reached on the wavenumber, together with an accuracy of $1 \%$ on its imaginary part, with only 5 or 6 points per wavelength with the 4th order SEM, while more than 9 points are necessary with the 2nd order FEM. Thus for the same level of accuracy in the dispersion curves of Fig. 3, a 2nd order FEM mesh should roughly have an element size $9 / 5$ finer than the 4 th order mesh, resulting in $(9 / 5)^{2}$ times more dofs (that is to say about 


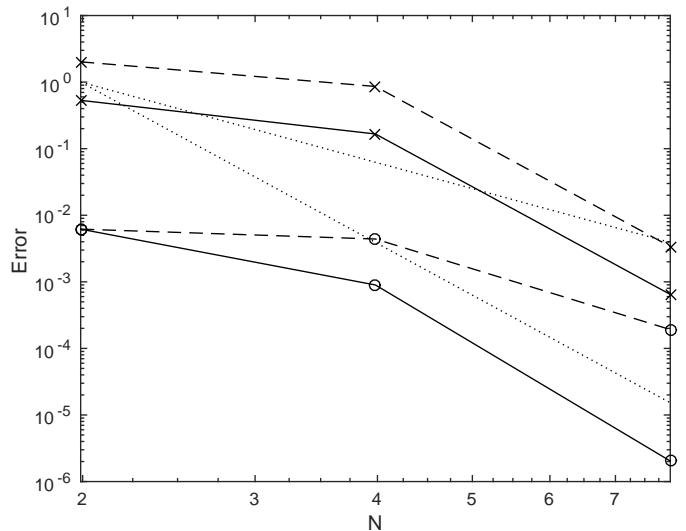

(a)

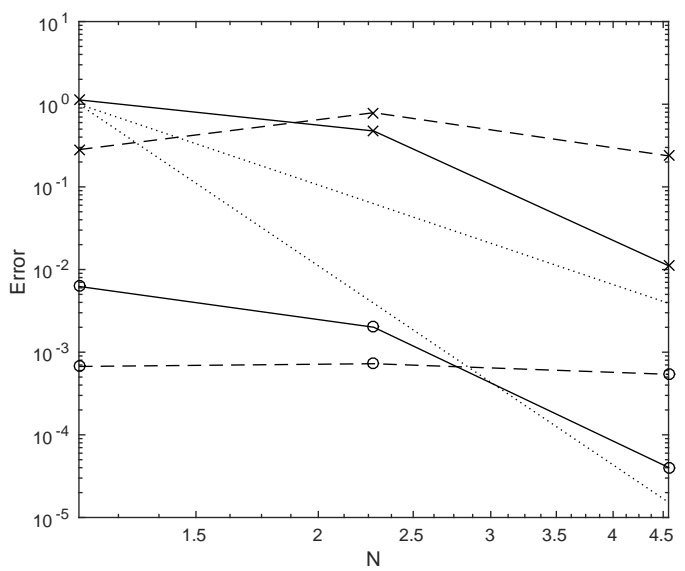

(b)

Figure 4: Numerical convergence for: (a) the $L(0,8)$ mode at $13.1 \mathrm{MHz}-\mathrm{mm}$, (b) the $L(0,12)$ mode at $22.9 \mathrm{MHz}-\mathrm{mm}$. Continuous lines: 4th order SEM, Dashed lines: 2nd order FEM. Circles: relative error $\left|k-k_{r}\right| /\left|k_{r}\right|$, Crosses: relative error for the imaginary part of the wavenumber. Dotted lines indicate the theoretical convergence rates of slope $s=-4$ and $s=-8$.

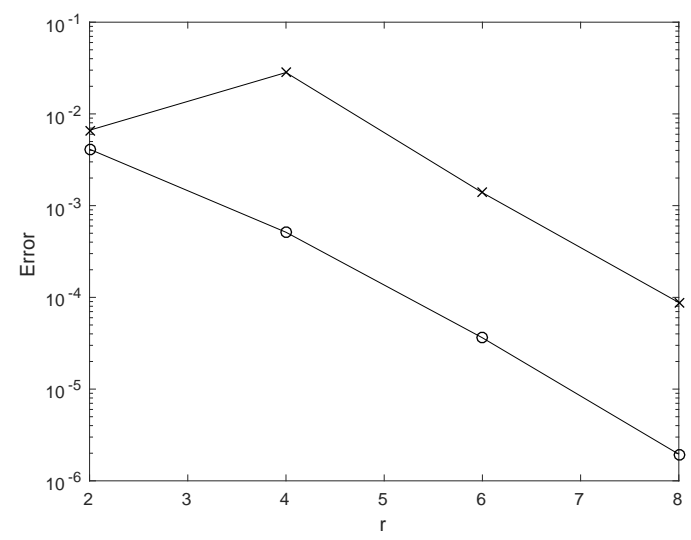

Figure 5: Relative errors as a function of $r$ ( $p$-convergence check) for the $L(0,4)$ mode at $5.3 \mathrm{MHz}$-mm. Same legend as Fig. 4.

130, 000 dofs, here).

Finally, the consistency of implementation of the SEM method is checked through $p$-refinement. The initial mesh is set as Mesh 1 of Tab. 3 for $r=2$. A $p$-refinement of this mesh is performed for $r=4,6$ and 8 , yielding $h / a=16,24$ and 32 respectively. The reference solution is the numerical solution computed from an 8 th order mesh with $h / a=64$. Figure 5 shows the semi-log plots of the errors as a function of the element order $r$ for the $L(0,4)$ mode at its minimum attenuation $\left(5.3 \mathrm{MHz}-\mathrm{mm}, k_{r}=5.2004+0.0636 \mathrm{i}\right)$. As expected exponential convergence is achieved with respect to $r$ : the curves exhibit a linear decrease with $r$ (except for the lowest order). It is noteworthy that the exponential convergence turns out to hold for the imaginary part of the wavenumber also, which confirms the trends previously observed in Fig. 4.

\subsection{Weak contrast of material impedance}

Low-leakage modes of compressional type actually have a motion that tends to vanish near the interface [3] and are less sensitive to the external medium. However, one expects that this sensitivity to the external medium will increase as the contrast of material impedance is getting weaker (the limiting case being identical 


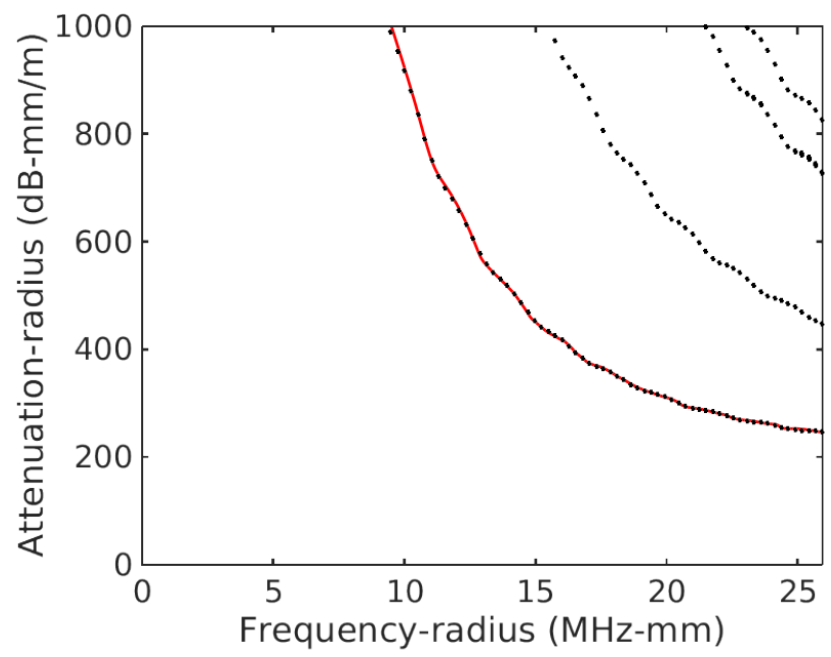

Figure 6: Attenuation dispersion curves for a weak contrast of material impedance, computed with the 4th order SEM mesh of Fig. 1 (39267 dofs). Red continuous line: results of Ref. [3].

materials). Hence, the feasibility of the proposed numerical approach needs to be demonstrated for a weak contrast of material impedance also.

The same test case as before is considered except that the external medium is now replaced with cast iron, which has properties close to steel (see Table 1). This test case is taken from Ref. [3]. The SEM mesh and the PML parameters are identical to those of Sec. 3.2.

Figure 6 shows the attenuation dispersion curves obtained from the PML waveguide formulation. Setting a weak contrast of material impedance strongly changes the dispersion curves. Also shown in Fig. 6 are the analytical results of Ref. [3] (calculated from the software Disperse). A close agreement is found, which show the feasibility of the approach for a wide range of material contrast.

Compared to the previous test case, the minimum attenuation occurs for a low-order mode, the $\mathrm{L}(0,2)$ mode, which is due to a mode crossing behavior of its phase velocity (in the previous test case, such a behavior also occurs for the high-order L(0,12) mode). As explained in Ref. [3], the phase velocity of the lowest attenuation mode indeed remains above the steel bulk longitudinal velocity and crosses the curves of the higher order modes. This mode crossing phenomenon is well reproduced by the numerical method.

As a side remark, the case of an external medium having faster bulk velocities than in the core is not discussed in this paper because it leads to the existence of trapped waves [46] and it seldom occurs for NDE applications (the external medium is usually made of soft materials). Furthermore as opposed to leaky modes, it is noteworthy that trapped modes do not loose energy in the external medium: they naturally decrease in the transverse direction so that their computation is less critical than leaky modes (trapped modes can yet be accurately computed with an elastic PML waveguide formulation also [26]).

\subsection{A non-canonical example}

As a final example, a square steel bar embedded in cement grout is considered. No analytical solution is available in this case. This example has been studied in Ref. [16] in a low-frequency regime. Here, a high-frequency analysis is performed in order to identify the possibility of low-leakage modes. The material properties, the PML and solving parameters are identical to those of Sec. 3.2 except that 8th order spectral elements are used to discretize the geometry (81-node quadrangles). This order has been set to achieve an accuracy of approximately $1 \%$ for the lowest imaginary part of the eigenvalue with approximately 5 points per wavelength as before (the reference solution being obtained from a refined mesh having twice as many points). The element order must be greater than in the cylindrical case because of the singularities at re-entrant corners (i.e. corners at the steel-grout interface). Note that in this case, it could have been 


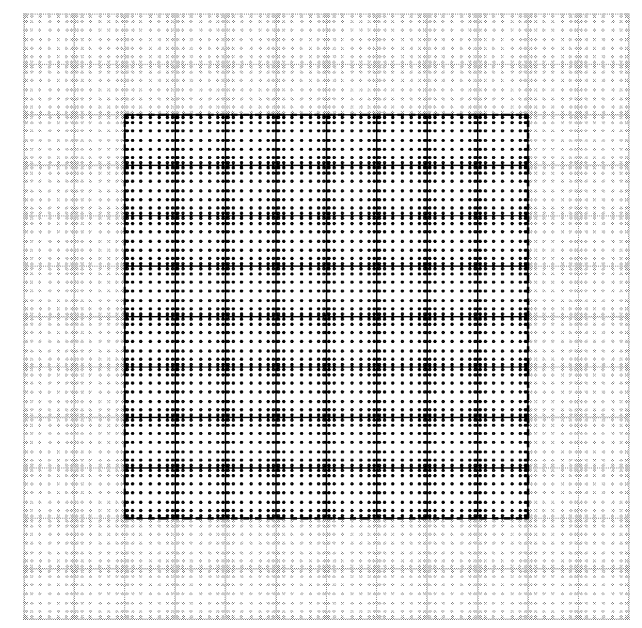

Figure 7: Discretization of an embedded square bar waveguide with the 8th order SEM (28995 dofs). Black: square bar (steel), gray: embedment (cement grout). Embedment and PML regions coincide with each other.

\begin{tabular}{lcc}
\hline \hline Mode & Frequency-radius (MHz-mm) & Attenuation-radius (dB-mm/m) \\
\hline$L_{8}$ & $12.7(12.8)$ & $207(207)$ \\
$L_{9}$ & $14.6(14.8)$ & $184(181)$ \\
$L_{10}$ & $16.7(16.9)$ & $166(165)$ \\
$L_{11}$ & $18.7(18.9)$ & $151(156)$ \\
$L_{12}$ & $22.5(23.1)$ & $147(149)$ \\
\hline \hline
\end{tabular}

Table 4: Numerical results obtained with 8th order SEM at the attenuation minima of five modes. In parentheses: numerical results with 2nd order FEM.

more advantageous to use a $h p$-adaptive refinement [47] or to use a radical mesh [48] rather than equispaced mesh, but such meshing strategies are left beyond the scope of this paper for simplicity.

Note that $a$ now denotes the half-width of the steel square bar. Figure 7 shows the mesh (28995 dofs). With 4 spectral elements of order 8 along the bar half-width, this mesh roughly satisfies a 5 point per wavelength criterion. Note that the PML interface position is still set to $d_{\alpha}=a$ (the interface is hence now stuck to the square bar).

Figure 8 shows the energy velocity and attenuation dispersion curves computed with 8th order elements. From a physical point of view, it is noteworthy that low-leakage modes occur in square bars similarly to cylindrical bars. The attenuation minima coincide with energy velocity maxima and the attenuation minima decrease up to a certain frequency (here, $22.5 \mathrm{MHz}-\mathrm{mm}$ ) and then increase due to the predominance of viscoelastic attenuation in the limit of very high frequency. Besides, these low-leakage modes are still found near the longitudinal bulk wavenumber of the steel core, $k_{0}=\omega / c_{l_{0}}$.

It has been checked by the inspection of mode shapes that these low-leakage modes are of longitudinal type. These modes are denoted as $L_{n}$ following the labeling used for free square bars in Ref. [49]. Similarly to cylindrical bars [3], the low-leakage property of longitudinal modes indeed results from the weakness of the acoustic field at the core-embedment interface. Figure 9 shows the example of the $L_{12}$ axial displacement in the square bar, as well as that of the $L(0,12)$ mode in the cylindrical bar considered in Sec. 3.2. Both mode shapes clearly tend to vanish at the core-embedment interface.

Table 4 gives some numerical results for the attenuation minima and their corresponding frequencies. These values remain quite close to those obtained for a cylindrical bar in Table 2 .

For comparison, Fig. 8 and Table 4 also provide the results computed with the 2nd order FEM (mesh not shown for paper conciseness). Each quadrangle of the 8th order SEM mesh has been subdivided into 16 


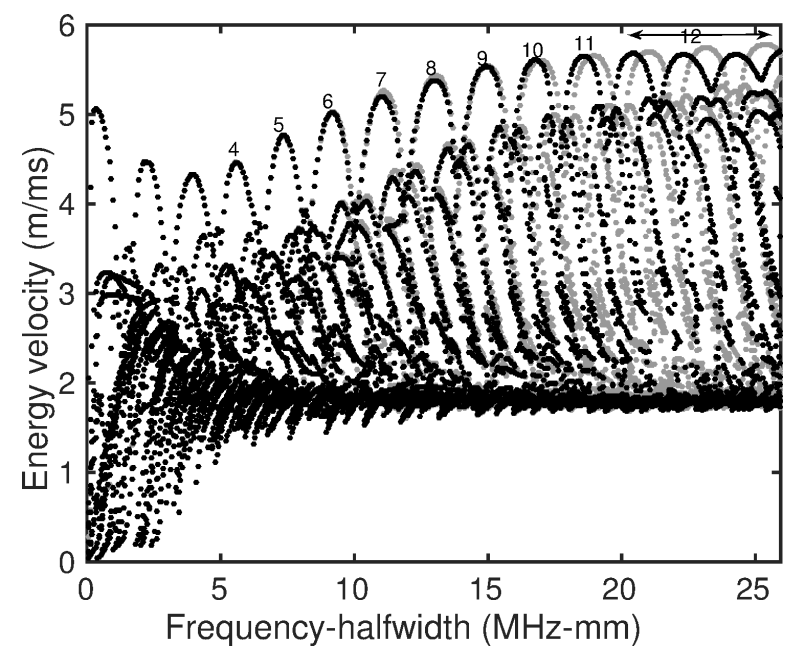

(a)

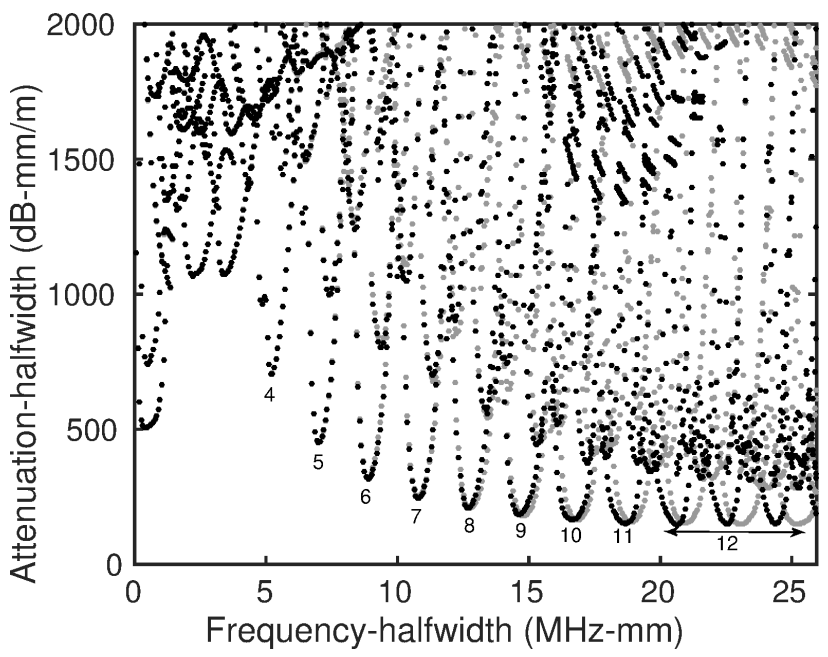

(b)

Figure 8: Dispersion curves of $L_{n}$ modes in an embedded square bar: (a) energy velocity, (b) attenuation. Black: 8th order SEM results, gray: 2nd order FEM.

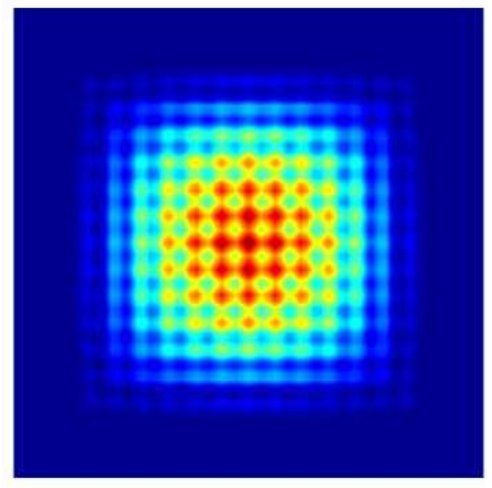

(a)

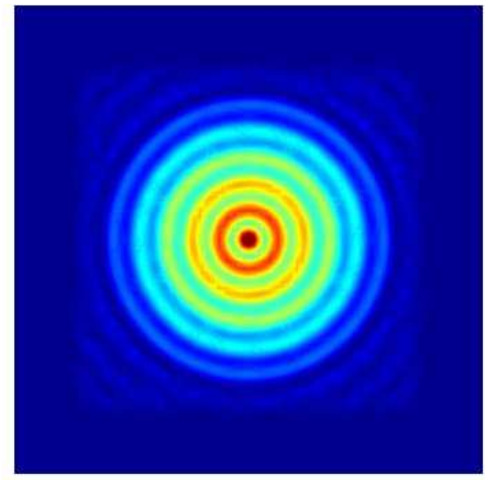

(b)

Figure 9: Axial displacement modulus of: (a) the $L_{12}$ mode at $22.5 \mathrm{MHz}-\mathrm{mm}$ in a square bar, (b) the $L(0,12)$ mode at 22.9 $\mathrm{MHz}-\mathrm{mm}$ in a cylindrical bar. 
quadrangles in order to generate exactly the same number of dofs between both meshes. It can be observed that the 2nd order FEM mesh fails to approximate the modal behavior of highest low-leakage modes and that the performance of 2 nd order elements appears to be worse than in the cylindrical case. This can be attributed to the fact that the solutions for a square bar are not as smooth as those for a cylindrical bar due to the corner effects of the square geometry.

From a physical point of view, these results show that the properties of low-leakage modes observed in the literature for cylindrical bars are hence likely to hold for other types of geometry. In particular, it can be inferred that low-leakage modes of longitudinal type still exist in waveguides of convex regular polygonal cross-section of rotational symmetry of order $n \geq 4$ (owing to the fact that squares and circles can be viewed as extreme cases, corresponding to $n=4$ and $n \rightarrow \infty$ respectively).

\section{Conclusion}

A waveguide formulation has been proposed for the high-frequency modeling of open solid waveguides of arbitrary shapes. The formulation uses a PML technique to account for the infinite surrounding media and is based on a SEM discretization of the cross-section to achieve an acceptable accuracy with a reduced number of dofs in the high-frequency regime. By focusing on low-leakage modes, the eigensolver shift around the longitudinal bulk wavenumber of the core allows reducing the number of modes to compute. The PML technique has the advantage of not modifying the structure of the eigensystem, which can remain linear as for closed waveguides. Although the computational cost increases compared to closed waveguides because of the necessity of meshing part of the exterior region, the PML thickness can be quite small (typically half the characteristic length of the core in this paper).

The PML waveguide formulation has shown to perform well in the test case of an embedded cylindrical bar. Based on this formulation, the SEM expectedly outperforms the FEM. It allows to readily handle high dimensionless frequency, up to 50 in the paper results. As already found in the literature for cylindrical bars, it has been observed that the attenuation minima of the $L(0, n)$ modes almost coincide with their energy velocity maxima. The successive attenuation minima decrease up to a certain frequency and then increase slowly due to the counteracting effects of viscoelasticity and leakage. Besides, a close agreement has been found with literature results obtained in the case of a weak contrast of impedance, which show the feasibility of the approach for a wide range of material contrast.

Finally, numerical results have been obtained for an embedded square bar, which has no analytical solution. These results show that low-leakage modes still occur near the bulk compressional phase velocity of the core, with the same dispersive features as their counterparts in cylinders. The properties of lowleakage modes in cylindrical bars are hence likely to hold for other types of geometry, including in particular convex regular polygonal cross-section of rotational symmetry.

\section{Acknowledgements}

The author wishes to thank the anonymous reviewers for their helpful comments.

\section{Appendix A. Structure of the matrix $K_{3}$ with SEM}

Applying the GLL quadrature to the expression of $\mathbf{K}_{3}^{e}$ given in Eq. (9) yields:

$$
\left.\mathbf{K}_{3}^{e} \simeq \sum_{i=1}^{r+1} \sum_{j=1}^{r+1} \omega_{i} \omega_{j}\left(\mathbf{N}^{e \mathrm{~T}} \mathbf{L}_{3}^{\mathrm{T}} \mathbf{C} \mathbf{L}_{3} \mathbf{N}^{e} \gamma_{1} \gamma_{2}\left|\operatorname{det} \mathbf{J}^{e}\right|\right)\right|_{(\xi, \eta)=\left(\xi_{i}, \eta_{j}\right)}
$$

or equivalently:

$$
\left.\mathbf{K}_{3}^{e} \simeq \sum_{l=1}^{n} w_{l}\left(\mathbf{N}^{e \mathrm{~T}} \mathbf{L}_{3}^{\mathrm{T}} \mathbf{C L}_{3} \mathbf{N}^{e} \gamma_{1} \gamma_{2}\left|\operatorname{det} \mathbf{J}^{e}\right|\right)\right|_{\boldsymbol{\xi}=\boldsymbol{\xi}_{l}}
$$


where $n=(r+1)^{2}$ is the number of nodes of the quadrangle, $w_{l}=\omega_{i} \omega_{j}$, $\boldsymbol{\xi}$ denotes the point of coordinates $(\xi, \eta)$ in the reference space and $l=l(i, j)$ is defined as $l=i+(r+1)(j-1)$.

The form of the interpolation matrix $\mathbf{N}^{e}$, as conventionally obtained from FEM, is:

$$
\mathbf{N}^{e}=\left(\begin{array}{llll}
N_{1}^{e} \mathbf{I} & N_{2}^{e} \mathbf{I} & \ldots & N_{n}^{e} \mathbf{I}
\end{array}\right)
$$

where $\mathbf{I}$ is the three-by-three identity matrix.

Expanding the matrix products inside (A.2) allows to get the following expression:

$$
\mathbf{N}^{e \mathrm{~T}} \mathbf{L}_{3}^{\mathrm{T}} \mathbf{C L}_{3} \mathbf{N}^{e}=\left(\begin{array}{cccc}
\mathbf{a}_{11} & \mathbf{a}_{12} & \ldots & \mathbf{a}_{1 n} \\
\mathbf{a}_{21} & \mathbf{a}_{22} & \ldots & \mathbf{a}_{2 n} \\
\vdots & \vdots & \ddots & \vdots \\
\mathbf{a}_{n 1} & \mathbf{a}_{n 2} & \ldots & \mathbf{a}_{n n}
\end{array}\right)
$$

with:

$$
\mathbf{a}_{p q}=\left(\begin{array}{lll}
C_{55} & C_{56} & C_{35} \\
C_{56} & C_{66} & C_{36} \\
C_{35} & C_{36} & C_{33}
\end{array}\right) N_{p}^{e} N_{q}^{e}
$$

where $p=1, \ldots, n$ and $q=1, \ldots, n$

The two-dimensional interpolants $N_{p}^{e}(p=1, . ., n)$ can be decomposed as the product between the onedimensional interpolants $h_{i}^{e}: N_{p}^{e}(\xi, \eta)=h_{i}^{e}(\xi) h_{j}^{e}(\eta)$. From the SEM property (14), one gets the following relation:

$$
N_{p}^{e}\left(\boldsymbol{\xi}_{l}\right)=\delta_{l p}
$$

From Eqs. (A.2), (A.4)-(A.6), the matrix $\mathbf{K}_{3}^{e}$ finally becomes:

$$
\mathbf{K}_{3}^{e}=\left(\begin{array}{cccc}
\left.w_{1}\left(\mathbf{a}_{11} \gamma_{1} \gamma_{2}\left|\operatorname{det} \mathbf{J}^{e}\right|\right)\right|_{\boldsymbol{\xi}=\boldsymbol{\xi}_{1}} & 0 & \ldots & 0 \\
0 & \left.w_{2}\left(\mathbf{a}_{22} \gamma_{1} \gamma_{2}\left|\operatorname{det} \mathbf{J}^{e}\right|\right)\right|_{\boldsymbol{\xi}=\boldsymbol{\xi}_{2}} & \ldots & 0 \\
\vdots & \vdots & \ddots & \vdots \\
0 & 0 & \ldots & \left.w_{n}\left(\mathbf{a}_{n n} \gamma_{1} \gamma_{2}\left|\operatorname{det} \mathbf{J}^{e}\right|\right)\right|_{\boldsymbol{\xi}=\boldsymbol{\xi}_{n}}
\end{array}\right)
$$

which shows that $\mathbf{K}_{3}^{e}$ is block diagonal with square blocks of dimension three. From Eq. (A.5), $\mathbf{K}_{3}^{e}$ is diagonal if $C_{35}=C_{36}=C_{56}=0$, i.e. for isotropic materials and transversely isotropic materials.

\section{References}

[1] M. J. S. Lowe, Plate waves for the ndt of diffusion bonded titanium, Ph.D. thesis, Mechanical Engineering Department, Imperial College London (1992).

[2] B. Pavlakovic, Leaky guided ultrasonic waves in ndt, Ph.D. thesis, Mechanical Engineering Department, Imperial College London (1998).

[3] B. N. Pavlakovic, M. J. S. Lowe, P. Cawley, High-frequency low-loss ultrasonic modes in embedded bars, Journal of Applied Mechanics 68 (2001) 67-75.

[4] M. D. Beard, M. J. S. Lowe, Non-destructive testing of rock bolts using guided ultrasonic waves, International Journal of Rock Mechanics and Mining Sciences 40 (2003) 527-536.

[5] T. Hayashi, W.-J. Song, J. L. Rose, Guided wave dispersion curves for a bar with an arbitrary cross-section, a rod and rail example, Ultrasonics 41 (2003) 175-183.

[6] I. Bartoli, A. Marzani, F. Lanza di Scalea, E. Viola, Modeling wave propagation in damped waveguides of arbitrary cross-section, Journal of Sound and Vibration 295 (2006) 685-707.

[7] F. Treyssède, Elastic waves in helical waveguides, Wave Motion 45 (2008) 457 - 470.

[8] S. Finnveden, M. Fraggstedt, Waveguide finite elements for curved structures, Journal of Sound and Vibration 312 (2008) 644-671.

[9] P. Sabiniarz, W. Kropp, A waveguide finite element aided analysis of the wave field on a stationary tyre, not in contact with the ground, Journal of Sound and Vibration 329 (2010) 3041-3064

[10] G. R. Liu, J. D. Achenbach, Strip element method to analyze wave scattering by cracks in anisotropic laminated plates, Journal of Applied Mechanics 62 (1995) 607-613. 
[11] H. Gravenkamp, H. Man, C. Song, J. Prager, The computation of dispersion relations for three-dimensional elastic waveguides using the scaled boundary finite element method, Journal of Sound and Vibration 332 (2013) $3756-3771$.

[12] H. Gravenkamp, C. Birk, C. Song, Simulation of elastic guided waves interacting with defects in arbitrarily long structures using the scaled boundary finite element method, Journal of Computational Physics 295 (2015) 438-455.

[13] R. E. Collin, Field Theory of Guided Waves, IEEE Press, 1991.

[14] M. Castaings, M. Lowe, Finite element model for waves guided along solid systems of arbitrary section coupled to infinite solid media, Journal of the Acoustical Society of America 123 (2008) 696-708.

[15] Z. Fan, M. J. S. Lowe, M. Castaings, C. Bacon, Torsional waves propagation along a waveguide of arbitrary cross section immersed in a perfect fluid, Journal of the Acoustical Society of America 124 (2008) 2002-2010.

[16] M. Mazzotti, I. Bartoli, A. Marzani, E. Viola, A coupled SAFE-2.5D BEM approach for the dispersion analysis of damped leaky guided waves in embedded waveguides of arbitrary cross-section, Ultrasonics 53 (2013) $1227-1241$.

[17] M. Mazzotti, I. Bartoli, A. Marzani, E. Viola, A 2.5D boundary element formulation for modeling damped waves in arbitrary cross-section waveguides and cavities, Journal of Computational Physics 248 (2013) 363-382.

[18] M. Mazzotti, I. Bartoli, A. Marzani, Ultrasonic leaky guided waves in fluid-coupled generic waveguides: hybrid finiteboundary element dispersion analysis and experimental validation, Journal of Applied Physics 115 (2014) 143512.

[19] H. Gravenkamp, C. Birk, J. Van, Modeling ultrasonic waves in elastic waveguides of arbitrary cross-section embedded in infinite solid medium, Computer \& Structures 149 (2015) 61-71.

[20] T. Hayashi, D. Inoue, Calculation of leaky lamb waves with a semi-analytical finite element method, Ultrasonics 54 (2014) 1460-1469.

[21] A. C. Hladky-Hennion, P. Langlet, R. Bossut, M. De Billy, Finite element modelling of radiating waves in immersed wedges, Journal of Sound and Vibration 212 (1998) 265-274.

[22] R. Jedidi, R. Pierre, High-order finite-element methods for the computation of bending loss in optical waveguides, Journal of Lightwave Technology 25 (2007) 2618-2630.

[23] Y. Ould Agha, F. Zolla, A. Nicolet, S. Guenneau, On the use of PML for the computation of leaky modes. an application to microstructured optical fibres, The International Journal for Computation and Mathematics in Electrical and Electronic Engineering 27 (2008) 95-109.

[24] A. S. Bonnet-BenDhia, B. Goursaud, C. Hazard, A. Prieto, A multimodal method for non-uniform open waveguides, International Congress on Ultrasonics, Physics Procedia 3 (2010) 497-503.

[25] A. Pelat, S. Felix, V. Pagneux, A coupled modal-finite element method for the wave propagation modeling in irregular open waveguides, Journal of the Acoustical Society of America 129 (2011) 1240-1249.

[26] F. Treyssède, K. L. Nguyen, A.-S. Bonnet-BenDhia, C. Hazard, Finite element computation of trapped and leaky elastic waves in open stratified waveguides, Wave Motion 51 (2014) 1093-1107.

[27] K. L. Nguyen, F. Treyssède, C. Hazard, Numerical modeling of three-dimensional open elastic waveguides combining semi-analytical finite element and perfectly matched layer methods, Journal of Sound and Vibration 344 (2015) 158-178.

[28] S. Kim, J. E. Pasciak, The computation of resonances in open systems using a perfectly matched layer, Mathematics of Computation 78 (2009) 1375-1398.

[29] S. Kim, Cartesian PML approximation to resonances in open systems in $\mathbb{R}^{2}$, Applied Numerical Mathematics 81 (2014) $50-75$.

[30] H. Gravenkamp, C. Birk, C. Song, Computation of dispersion curves for embedded waveguides using a dashpot boundary condition, Journal of the Acoustical Society of America 135 (2014) 1127-1138.

[31] A. Patera, A spectral element method for fluid dynamics: laminar flow in a channel expansion, Journal of Computational Physics 54 (1984) 468-488.

[32] G. Seriani, E. Priolo, Spectral element method for acoustic wave simulation in heterogeneous media, Finite Elements in Analysis and Design 16 (1994) 337-348.

[33] D. Komatitsch, J. Tromp, Introduction to the spectral element method for three-dimensional seismic wave propagation, Geophysical Journal International 139 (1999) 806-822.

[34] A. B. Andreev, V. A. Kascieva, M. Vanmaele, Some results in lumped mass finite-element approximation of eigenvalue problems using numerical quadrature formulas, Journal of Computational and Applied Mathematics 43 (1992) $291-311$.

[35] C. Pozrikidis, Finite and Spectral Element Method Using Matlab, Second Edition, CRC Press, Boca Raton, 2014.

[36] T. H. Vu, A. J. Deeks, Use of higher-order shape functions in the scaled boundary finite element method, International Journal of Numerical Methods in Engineering 65 (2006) 1714-1733.

[37] D. Komatitsch, R. Martin, J. Tromp, Wave propagation in 2-d elastic media using a spectral element method with triangles and quadrangles, Journal of Computational Acoustics 9 (2001) 703-718.

[38] F. Tisseur, K. Meerbergen, The quadratic eigenvalue problem, SIAM Review 43 (2001) 235-286.

[39] R. Lehoucq, D. Sorensen, C. Yang, ARPACK User's Guide: Solution of Large Scale Eigenvalue Problems with Implicitly Restarted Arnoldi Methods, SIAM, Philadelphia, PA, 1998.

[40] V. Baronian, Couplage des méthodes modale et éléments finis pour la diffraction des ondes élastiques guidées : Application au contrôle non destructif (coupling of modal and finite elements methods for the diffraction of guided elastic waves application to non destructive testing, in french), Ph.D. thesis, Ecole Polytechnique (2009).

[41] M. Beard, Guided wave inspection of embedded cylindrical structures, Ph.D. thesis, Mechanical Engineering Department, Imperial College London (2002).

[42] A. Bernard, M. J. S. Lowe, M. Deschamps, Guided waves energy velocity in absorbing and non-absorbing plates, Journal of the Acoustical Society of America 110 (2001) 186-196.

[43] C. Geuzaine, J.-F. Remacle, Gmsh: a three-dimensional finite element mesh generator with built-in pre- and postprocessing facilities, International Journal for Numerical Methods in Engineering 79 (2009) 1309-1331. 
[44] D. Komatitsch, S. Tsuboi, J. Tromp, The spectral-element method in seismology, in: A. Levander, G. Nolet (Eds.), Seismic Earth: Array Analysis of Broadband Seismograms, Vol. 157 of Geophysical Monograph, American Geophysical Union, Washington DC, USA, 2005, pp. 205-228.

[45] B. N. Pavlakovic, M. J. S. Lowe, D. N. Alleyne, P. Cawley, Disperse: A general purpose program for creating dispersion curves, in: Review of Progress in Quantitative NDE, Vol. 16, 1997, pp. 185-192.

[46] R. N. Thurston, Elastic waves in rods and clad rods, Journal of the Acoustical Society of America 64 (1978) 1-37.

[47] I. Babuska, M. Suri, The p and h-p versions of the finite element method: basic principles and properties, SIAM Review 36 (1994) 578-632.

[48] P. Vos, S. Sherwin, R. Kirby, From h to p efficiently: Implementing finite and spectral/hp element methods to achieve optimal performance for low- and high-order discretisations, Journal of Computational Physics 229 (2010) 5161-5181.

[49] A. Gunawan, S. Hirose, Boundary element analysis of guided waves in a bar with an arbitrary cross-section, Engineering Analysis with Boundary Elements 29 (2005) 913-924. 